\title{
Stage IV Uterine Corpus Endometrial Stromal Sarcoma AJCC v8
}

National Cancer Institute

\section{Source}

National Cancer Institute. Stage IV Uterine Corpus Endometrial Stromal Sarcoma A/CC v8. NCI Thesaurus. Code C139891.

Stage IV includes: IVA: T4, Any N, M0; IVB: Any T, Any N, M1. T4: Uterine corpus endometrial stroma sarcoma with tumor invading bladder or rectum. M0: No distant metastasis. M1: Distant metastasis (excluding adnexa, pelvic, and abdominal tissues). (from AJCC 8th Ed.) 